\title{
NUMERICAL SEMIGROUPS BOUNDED BY A CYCLIC MONOID
}

\author{
M. A. Moreno-Frías And J. C. Rosales
}

Abstract. We will say that a numerical semigroup $S$ is bounded by a cyclic monoid if there exist integer numbers $0 \leqslant \alpha<\beta$ such that $S=\{x \in \mathbb{N} \mid k \alpha<x<k \beta$ for some $k \in \mathbb{N}\} \cup\{0\}$. The goal of this work is to study this kind of numerical semigroups. In particular, we will determine important invariants of them such as multiplicity, embedding dimension, Frobenius number and genus.

Mathematics subject classification (2020): Primary 20M14; Secondary 11D07.

Keywords and phrases: $\mathscr{A} \mathscr{C}$-semigroup, cyclic monoid, diophantine inequality, embedding dimension, Frobenius number, genus, MED-semigroup, multiplicity, numerical semigroup, PM-semigroup.

\section{REFERENCES}

[1] R. APÉRY, Sur les branches superlinéaires des courbes algébriques, C. R. Acad. Sci. Paris 222 (1946), 1198-2000.

[2] P. T. Bateman, Remark on a recent note on linear forms, Amer. Math. Monthly 65 (1958), 517-518.

[3] D. D. GRANT, On linear forms whose coefficientes are in arithmetic progression, Israel J. Math. 15 (1973), 204-209.

[4] J. B. Roberts, Note on linear forms, Proc. Amer. Math. Soc. 7 (1956), 465-469.

[5] A. M. Robles-PÉREZ AND J. C. Ros Ales, Numerical semigroups in a problem about cost-effective transport, Forum Math. 29 (2017), 329-345.

[6] J. C. Rosales And P. A. García-SÁncheZ, Numerical Semigroups, Developments in Mathematics, Vol. 20, Springer, New York, 2009.

[7] J. C. Rosales and J. M. Urbano-Blanco, Opened modular numerical semigroups, J. Algebra 306, (2006), 368-377.

[8] J. C. Rosales, P. A. García-Sánchez, J. I. García-García and J. M. Urbano-Blanco, Proportionally modular diophantine inequalities, J. Number Theory 103, (2003), 281-294.

[9] J. C. Rosales, P. A. García-SÁnchez, J. I. García-García And M. B. Branco, Numerical semigroups with maximal embedding dimension, Int. J. Commut. Ring 2, (2003), no. 1, 47-53.

[10] J. C. Rosales, P. A. García-SÁnchez And J. M. Urbano-Blanco, The set of solutions of a proportionally modular Diophantine inequality, J. Number Theory 128, (2008), 453-467.

[11] E. S. Selmer, On the linear diophantine problem of Frobenius, J. Reine Angew. Math. 293/294 (1977), 1-17.

[12] A. TRIPATHI, The Frobenius problem for modified arithmetic progressions, Journal of Integer Sequences 16 (2013), Article 13.7.4. 